\title{
Prospective motion correction and selective reacquisition using volumetric navigators for vessel-encoded arterial spin labelling dynamic angiography
}

Robert Frost ${ }^{1}$, Aaron T. Hess ${ }^{2}$, Thomas W. Okell ${ }^{1}$, Michael A. Chappell1,3, M. Dylan Tisdall ${ }^{4,5}$, André J. W. van der Kouwe ${ }^{4,5}$, Peter Jezzard ${ }^{1}$

1. FMRIB Centre, Nuffield Department of Clinical Neurosciences, University of Oxford, Oxford, UK

2. Oxford Centre for Clinical Magnetic Resonance Research, Division of Cardiovascular Medicine, Radcliffe Department of Medicine, University of Oxford, Oxford, UK

3. Institute of Biomedical Engineering, University of Oxford, Oxford, UK

4. A. A. Martinos Centre for Biomedical Imaging, Massachusetts General Hospital, Charlestown, MA, USA

5. Dept of Radiology, Harvard Medical School, Boston, MA, USA

Word count: 4900

Running title: Volumetric EPI navigators for VEPCASL dynamic angiography

Address for Correspondence:

Robert Frost, D.Phil.

FMRIB Centre

John Radcliffe Hospital

Oxford

OX3 9DU

UK

Tel: +44 (0)1865222488

Fax: +44 (0)1865 222717

Email: robert.frost@ndcn.ox.ac.uk 


\begin{abstract}
Purpose: The aim of this study was to improve robustness to motion in a vesselencoded angiography sequence used for patient scans. The sequence is particularly sensitive to motion between imaging segments, which causes ghosting and blurring that propagates to the final angiogram.

Methods: Volumetric EPI navigators acquired in $275 \mathrm{~ms}$ were inserted after the imaging readout in a vessel-encoded pseudo-continuous arterial spin labelling (VEPCASL) sequence. The effects of movement between segments on the images were tested with phantom experiments. Deliberate motion experiments with healthy volunteers were performed to compare prospective motion correction (PMC) with reacquisition versus no correction.

Results: In scans without motion, the addition of the EPI navigator to the sequence did not affect the quality of the angiograms in comparisons with the original sequence. PMC and reacquisition improved the visibility of vessels in the angiograms compared to the scans without correction. The reacquisition strategy was shown to be important for complete correction of imaging artifacts. Conclusion: An effective method to correct motion in vessel-encoded angiography has been demonstrated. For reacquisition of 15 segments, the technique requires approximately 30 s of additional scanning ( 25\%).
\end{abstract}




\section{Introduction}

Vessel-selective dynamic angiography (1-5) has recently been demonstrated with vessel-encoded pseudo-continuous arterial spin labelling (VEPCASL) (6). This is a promising non-invasive technique for visualisation of vessel architecture and flow patterns, particularly for assessment of collateral blood flow in patients with stenoocclusive disease (7). Vessel-selective information is generally obtained with X-ray digital subtraction angiography, which has high temporal and spatial resolution but is invasive, uses ionising radiation and requires catheter repositioning to assess different arteries.

Angiography based on image subtraction or comparison is sensitive to motion between images due to the implicit assumption that the static tissue signal is identical across all the images. In the VEPCASL method, the vessels of interest are encoded over a series of images, resulting in an acquisition time on the order of minutes. The combination of images across this relatively long time period increases the likelihood of static tissue subtraction artifacts, particularly in disorientated patients, such as those with acute stroke. In our implementation, two-dimensional angiograms are generated from a single thick slice $(\sim 50 \mathrm{~mm})$. Therefore retrospective registration $(8,9)$ of in-plane movement between images is possible but through-plane motion cannot be corrected. Further, each of the constituent vessel-encoded images is acquired with a segmented readout to obtain high spatial and temporal resolution. Inter-segment motion during the acquisition of one of these images creates k-space inconsistencies leading to image ghosting or blurring artifacts, which in turn propagate to the final angiogram.

Strategies to correct motion-related artifacts in segmented or 3D acquisitions rely on having knowledge of the motion, which can be applied in real time to update the imaging coordinates (10-17) or retrospectively to correct segments of the k-space data (18-21) or using a combination of both, which has been shown to reduce accuracy requirements of the motion tracking (22). Retrospective corrections can 
estimate regions of k-space that were not acquired, but they are unable to deal with through-plane motion in the current single-slice case. Therefore prospective methods that detect motion and make appropriate adjustments of the RF phase and gradients in real time to measure signal in the prescribed volume are more relevant to the current study.

Prospective corrections have used external equipment (independent of the MR sequence) such as fiducial markers (23), active markers (RF coils) (24) and optical cameras (13). These can in principle apply high-frequency updates to all sequences, however they depend on accurate calibration and often require markers fixed to the subject, which can be inconvenient for patient scans, as well as challenging to achieve in practice. For example, an optical tracking marker must be rigidly attached to the subject so that its movement is well correlated with head movement and visible to the camera. Using MR information is therefore an appealing alternative, as no externally attached markers are needed, and techniques can be used and distributed relatively easily. Furthermore, the flexibility of MR has been shown to be useful for tracking internal organs and non-rigid motion (25) as well as offering other information such as $B_{0}$ field measurements for first-order shim updates in spectroscopy (16). MR-based methods track motion with "navigator" information (26), which is intrinsic to sequences that cover the centre of k-space in each segment such as PROPELLER and spiral trajectories $(27,28)$. Examples of navigator modules that can be added to the host sequence include orbital and cloverleaf $\mathrm{k}$ space trajectories $(14,29)$, repeatedly acquiring and filtering three orthogonal images (PROMO) (15) and low-resolution EPI images $(16,17)$. In these latter studies, the PROMO and EPI navigator modules were acquired once per TR in highresolution anatomical sequences with no increase in scan time. Both studies demonstrated the importance of reacquisition in 3D sequences for segments acquired before motion was detected (i.e., in the interval between navigators) $(15,17)$. 
This study used volumetric EPI navigators for accurate prospective motion correction (PMC) and selective reacquisition in a vessel-encoded dynamic angiography sequence applied to the cerebral arteries. Previous work (30) has shown that PMC with EPI navigators can improve the quality of pulsed ASL perfusion imaging using a segmented GRASE readout (31). Volumetric navigator PMC has also been demonstrated in an angiographic method based on MPRAGE T1weighted anatomical imaging (32). As mentioned above, this ASL angiography method is particularly sensitive to inter-segment motion and therefore the high accuracy of the volumetric navigators is desirable. The inter-segment motion artifacts were demonstrated with phantom experiments and deliberate motion tests with healthy volunteers were used to compare the angiograms acquired with PMC and selective reacquisition to those with no real-time correction.

\section{Methods}

\section{Navigator sequence and reacquisition}

All data were acquired using a 3 T Siemens Verio scanner using a 32-channel head receive coil (Siemens Healthcare, Erlangen, Germany). Volumetric EPI navigators $(16,17)$ were added after the imaging readout of a VEPCASL angiography sequence (1). This study used cardiac triggering and pre-saturation, as described in previous work (33), and a 2D RF-spoiled gradient-recalled echo imaging readout. A schematic sequence diagram is shown in Fig. 1. The navigator was a 3D EPI acquisition with 8 $\mathrm{mm}$ isotropic resolution acquired with the following parameters: $32 \times 32 \times 32$ matrix, isotropic $256 \mathrm{~mm}$ field of view (FOV), 6/8 slice partial Fourier, flip angle $=2^{\circ}$, TR/TE $=14 / 6 \mathrm{~ms}$, bandwidth $=4006 \mathrm{~Hz} /$ pixel, total navigator time $=275 \mathrm{~ms}$.

The angiography sequence used cardiac triggering to begin the pre-saturation of the imaging volume, which was followed by a $700 \mathrm{~ms}$ VEPCASL preparation and the segmented imaging readout. The VEPCASL pulse train consists of a series of $20^{\circ} \mathrm{RF}$ pulses with slice gradients to invert spins passing through the tagging plane 
combined with transverse gradients to induce selective labelling (1). The sequence used here is similar to previous studies (1) but has been optimised for use in a busy patient study - a 4-fold (approximately) scan time reduction was achieved by compromising on temporal resolution and using parallel imaging. Images were acquired with the following parameters: $1.0 \mathrm{~mm}$ in-plane resolution, $50 \mathrm{~mm}$ slice thickness, $224 \times 168$ matrix, 220×165 mm FOV, asymmetric (partial) echo to reduce flow artifacts, R-L phase-encoding direction, 6/8 phase partial Fourier, factor 2 GRAPPA acceleration with 24 reference lines (34), $10 \mathrm{k}$-space lines acquired per segment, 8 segments per image, $\mathrm{TR}=11 \mathrm{~ms}$, segment $\mathrm{TR}$ (temporal resolution) = $112 \mathrm{~ms}, \mathrm{TE}=6 \mathrm{~ms}$, flip angle $=10^{\circ}$, bandwidth $=152 \mathrm{~Hz} /$ pixel. The sequence acquired eight images sequentially with different VEPCASL encodings, identical to those described by Okell et al. (35), to enable angiograms arising from the four main brain-feeding arteries (the right and left internal carotid and vertebral arteries) to be reconstructed (6). The right (RICA) and left internal carotid (LICA) arteries and the right (RVA) and left vertebral arteries (LVA) were encoded in the following eight cycles: nonselective tag; nonselective control; tag right and control left; tag left and control right; tag anterior and control posterior; tag posterior and control anterior; tag RICA and LVA and control LICA and RVA; tag LICA and RVA and control RICA and LVA. The scan times of the navigator sequence without reacquisitions and with 15 reacquisitions were $\sim 2: 30 \mathrm{~min}$ and $\sim 3: 00 \mathrm{~min}$, respectively (cardiac cycle dependent).

In the current cardiac-triggered implementation, the addition of the navigator leads to a reduction in the possible number of cardiac phases or an increased acquisition time. The number of cardiac phases in the original sequence was determined by setting the VEPCASL labelling duration plus the acquisition window to be slightly less than two cardiac cycles. The number of phases is then given by dividing the acquisition window duration by the segment TR (112 ms). With an appropriately set acquisition window, the next cardiac trigger follows soon after the imaging readout has finished. Hence the addition of the navigator module would mean that the next cardiac trigger would be missed if the acquisition window, or equivalently the 
number of cardiac phases, were kept constant. Therefore, in this demonstration of the motion correction technique, the number of cardiac phases acquired was reduced by 2-3 to avoid an increased acquisition time. Typically 6 phases were acquired (rather than 8-9 in the original sequence) with the navigator sequence.

The navigator image reconstruction, registration and motion feedback can take 80$200 \mathrm{~ms}$, depending on the scanner's image reconstruction system (17). The $700 \mathrm{~ms}$ VEPCASL preparation allowed sufficient time after the navigator acquisition for these position updates to be generated using the PACE algorithm (11). PACE stops the real-time motion correction if the estimated motion exceeds $20 \mathrm{~mm}$ translation or 8 degrees rotation and the scan continues without updates to the imaging volume. This is to avoid inaccurate motion estimates caused by the head moving outside the navigator field of view. Imaging coordinates were updated before the imaging readout as indicated by the arrow in Fig. 1. The update consists of a pause where feedback is received, and a delay to allow time to generate the sequence instructions for the imaging readout following the pause. This update and delay process amounted to a $10 \mathrm{~ms}$ delay in total. The position of the VEPCASL labelling plane was not updated as the navigators measure head motion, which would not necessarily correspond to the non-rigid movement of the neck.

Between successive navigator acquisitions, a motion score was calculated from the six rigid-body movement parameters and linked to the imaging readout acquired between the two navigators. These scores were used to guide the selective reacquisition (at the end of the scan) by searching for the largest remaining motion score before every reacquisition (17). Originally-acquired data were only replaced by reacquired data if the reacquired motion score was lower than the original score (17). The final angiographic images are available on the scanner along with the navigator images for inspection of motion during the scan. The chosen motion score is computed from a combined angle magnitude given by 
$|\theta|=\left|\cos ^{-1}\left\{\frac{1}{2}\left[\cos \theta_{x} \cos \theta_{y}+\cos \theta_{x} \cos \theta_{z}+\cos \theta_{y} \cos \theta_{z}+\sin \theta_{x} \sin \theta_{y} \sin \theta_{z}-1\right]\right\}\right|$

where $\theta_{x}, \theta_{y}$ and $\theta_{z}$ are the respective Euler angles of the estimated rotation, expressed so that rotation is first applied around the x-axis, then around the y-axis and finally the z-axis. This angle is then used to calculate the maximum possible displacement of any point on a $64 \mathrm{~mm}$ sphere when rotated by $|\theta|$

$\Delta R=64 \sqrt{(1-\cos |\theta|)^{2}+(\sin |\theta|)^{2}}$

The final motion score is then given by

motion score $=\Delta R+\sqrt{\Delta_{x}^{2}+\Delta_{y}^{2}+\Delta_{z}^{2}}$

where $\Delta_{x}, \Delta_{y}$ and $\Delta_{z}$ are the estimated translations in $\mathrm{x}, \mathrm{y}$ and $\mathrm{z}$, respectively.

Angiography motion experiments

Comparisons of scans with "PMC off" to "PMC on" were made with the navigator version of the VEPCASL angiography sequence. The detected translations and rotations were checked to ensure that motion was comparable between the scans. Movements did not exceed the PACE limits of $20 \mathrm{~mm}$ translation or 8 degrees rotation mentioned above. In "PMC off" scans, the navigator was still played out, but the detected motion parameters were not applied to the angiography imaging readout.

Phantom experiments were performed to investigate artifacts caused by motion during the segmented acquisition. The phantom was moved by hand at the same point during image acquisition for the following cases: "PMC off", "PMC on", "PMC on 
and 1 reacquisition". Rotation about the $\mathrm{z}$ axis and translation in $\mathrm{z}$ were compared to scans with no movement.

Additionally, four healthy volunteers were scanned under an approved technical development ethics protocol. A conventional 3D multi-slab time-of-flight angiogram was acquired to select the VEPCASL labelling plane and establish the locations of the right and left internal carotid and vertebral arteries. A scan with "PMC on and 6 reacquisitions" during no deliberate motion was acquired as a reference. In two of the volunteers, the original sequence was also acquired with no deliberate motion for comparison with the navigator sequence.

Experiments were performed to assess three different motion conditions or types. For all conditions, movement started after four VEPCASL preparation and segment readout blocks, each of which is referred to as a TR:

motion A) - fast, discrete movements. Starting from a normal central position, subjects moved between the following positions every three TRs: head turned left, head looking down (chin to chest), head turned right, head tilted back, return to the central position, head turned left. The head remained turned left for the rest of the scan.

motion B) - slow, continuous movements. The same movement sequence as motion A above but moving between them slowly over the course of three TRs.

motion C) - fast, continuous movements. Alternating between shaking and nodding head for 10 TRs. Head in the central position for the remainder of the scan.

Examples of detected movements during the three motion conditions are shown in the left-hand column of Fig. 2. Each motion condition was repeated with "PMC off" compared to "PMC on and 15 reacquisitions". Volunteers were instructed to move as similarly as possible between the repeats of the same motion condition. The scan order, i.e., whether the "PMC off" scan was first or second in each motion condition, was randomised. 
An additional motion experiment with fewer movements was performed with one subject. Motions A and B were shortened and motion C was the same except that after the 10 TRs of motion, the subject remained in the head turned left position rather than the central position. As above, movement started after four TRs in all conditions:

motion $\mathrm{A}^{*}$ ) - fast, discrete movements. Starting from a normal central position, the subject moved between the following positions every three TRs: head turned left, head looking down (chin to chest), return to the central position, head turned left. The head remained turned left for the rest of the scan.

motion $\mathrm{B}^{*}$ ) - slow, continuous movements. The same movement sequence as motion $A^{*}$ above but moving between them slowly over the course of three TRs.

motion $\mathrm{C}^{*}$ ) - fast, continuous movements. Alternating between shaking and nodding head for 10 TRs. Head turned left for the remainder of the scan.

\section{Post-processing and data analysis}

All images from the angiography motion experiments were post-hoc registered to the volume acquired halfway through the scan (9). A Bayesian analysis method using a fast maximum a posteriori estimation of "global" parameters, such as the artery locations, was used to separate the signals arising from each brain-feeding artery in the angiographic data (4). Inferring the artery locations with realistic constraints allows for some movement of the subject after the initial planning. Note that the position of the labelling plane was not updated prospectively in this study. The temporal mean of the multiple cardiac phases was used for quality comparisons of the final angiograms.

The angiograms acquired during deliberate motion were given a quality score relative to each subject's angiogram acquired without deliberate motion. This quality score was intended to represent the visibility of signal arising from the four vessels that could be identified in the angiograms without deliberate motion. A score of 1 was given if the main branch of the vessel signal was visible (i.e., matching 
the no motion data) and a score of 2 was given if the distal, smaller vessels were also visible. These scores were assigned for the signal originating from the RICA, LICA and the combination of the RVA and LVA (which mix in the basilar artery). Hence possible scores ranged from 0 (no vessels visible) to a maximum of 6 (all the vessels visible). Mis-assignment of the arterial origin of the blood signal due to motion artefact, which is more frequent in the vertebral arteries and is evident in some of the results below, was not considered for these scores.

For all angiographic scans, the six detected motion parameters from each pair of navigators were used to generate motion scores using equations [1-3]. Examples of detected movements and the corresponding plots of the motion scores are shown in Fig. 2. A motion score threshold of $0.5 \mathrm{~mm}$ was determined empirically to decide whether movement relative to the last navigator was counted as a "significant" motion that would be a likely cause of artifacts. Scores above this threshold are marked as red crosses in Fig. 2 and have good correspondence with times when the subject was instructed to move. In each deliberate motion scan, the number of these significant motions was recorded and the mean score of the significant motions was calculated.

The effect of having fewer reacquisition TRs was also investigated retrospectively. Images from the "PMC on" data were reconstructed from the raw k-space data using all 15 , the first 10 , the first 5 or 0 reacquisitions.

\section{Results}

The temporal standard deviations of the six rigid-body motion estimates and the median motion scores from the two phantom scans without motion and the four invivo scans without intentional motion are provided in Table 1 . The phantom and invivo temporal metrics were generated from 7 and 70 relative motion estimates, respectively. Supporting Figure S1 provides an example of the estimated motion parameters from the scan without intentional motion from subject 1. 
Results of the phantom experiments showing the effect of movement during the segmented acquisition are shown in Fig. 3 along with example plots of the movement for each of the three motion scans. The movement plots for all scans are provided in Sup. Fig. S2. Severe artifacts are present when PMC is not used and although there is improvement in the "PMC on" scan, residual artifact is still apparent. This is due to the segment immediately before the navigator from which motion was detected. The detected motion is prospectively corrected before the next segment but the segment in the moved position is inconsistent with the others. When the segment that was acquired in the moved position is reacquired, the image artifacts are removed as shown in the fourth image column of Fig. 3.

Comparison of angiograms acquired without deliberate motion using the original sequence and the navigator (vNav) sequence are shown in Fig. 4. These show that the addition of the navigator, PMC and reacquisition do not have a detrimental effect on the data. Angiograms from the motion experiments in four subjects are shown in Fig. 5, demonstrating a general improvement in image quality with "PMC on and 15 reacquisitions", particularly for motion conditions A and C. Plots of the number of significant motions and the mean score of these motions are shown in Figs. 6a and $6 \mathrm{~b}$, respectively. These show the similarity of motion between scans and across subjects and therefore it is likely that differences in image quality can be attributed to the motion correction. The image quality scores of the angiograms are plotted in Fig. 6c. The red line in Fig. 6a marks the 15 reacquisitions threshold, which effectively corresponds to the number of significant motions that can be corrected. PMC with reacquisition scans with more than 15 significant motions are marked on Fig. 6c with red circles. These results suggest that having insufficient reacquisitions to correct all motion is the reason that there is no clear improvement with PMC and reacquisition for motion condition B. Overall, in the three motion conditions in each of the four subjects, the image quality score improved with "PMC on and 15 reacquisitions" in 9 of the 12 tests and was unchanged in 3 of the 12. 
Figure 7 demonstrates the effect of retrospectively using fewer reacquisitions in the image reconstruction. In this example, the motion $\mathrm{A}$ and $\mathrm{C}$ angiograms do not change appreciably between 15 and 10 reacquisitions but severely degrade with 5 or 0 . Using the $0.5 \mathrm{~mm}$ score threshold, the motion B acquisition contained 23 significant motions (see Fig. 6a) so the 15 reacquisitions used were insufficient, whereas there were only 12 and 13 motions in $A$ and $C$ respectively.

Results of the additional motion experiment are shown in Fig. 8. The movement conditions were shortened to ensure that there were fewer than 15 movements and condition $\mathrm{C}$ was altered slightly so that after the deliberate motion the subject's head was turned left for the remainder of the scan. Figure 8a shows that all scans with "PMC on and 15 reacquisitions" (image quality scores: $4\left(\mathrm{~A}^{*}\right), 5\left(\mathrm{~B}^{*}\right), 6\left(\mathrm{C}^{*}\right)$ ) have good agreement with the no motion scans and are an improvement on the "PMC off" scans (image quality scores: $\left.0\left(\mathrm{~A}^{*}\right), 3\left(\mathrm{~B}^{*}\right), 2\left(\mathrm{C}^{*}\right)\right)$. Motion logs of the deliberate motion scans are shown in Fig. 8b. The number of motions and the mean score of the motions are provided in Sup. Fig. S3 - the maximum number of motions in any of the scans was 12 . These results suggest that having sufficient reacquisitions to correct movements is indeed important. Also it suggests that the positioning after the motion period in $C$ (head in central position) and $C^{*}$ (head turned left) does not affect the angiograms.

\section{Discussion}

This study has demonstrated that the sensitivity of vessel-encoded angiography to image segmentation artifacts and image misalignment can be substantially improved with prospective motion correction. Image quality scores summarising the visibility of vessels were improved with PMC and reacquisition in $75 \%$ of the deliberate motion tests in four volunteers, with no improvement in the remainder. Further, the inclusion of EPI navigators to the sequence did not affect the quality of the angiograms. It was shown that the resulting image quality is strongly dependent on whether the number of segments that are reacquired exceeds the number of 
identified motion-corrupted segments. In the deliberate motion experiments, the subjects did not move intentionally during the reacquisition period and therefore all reacquisitions could replace motion-corrupted segments. Motion during the reacquisition period at the end of the scan will inevitably occur in patient scanning and hence reduce the effective number of movements that can be corrected. With approximately $30 \mathrm{~s}$ additional scan time for 15 reacquisitions, however, it is expected that the robustness of the scan to involuntary patient movements can be substantially improved.

The results of motion conditions A and A* shown in Figs. 4 and 8, which tested large, discrete changes of position, suggest that there is a particular sensitivity to this type of motion. The quality of the "no motion" scan could not be completely recovered with "PMC on and 15 reacquisitions" in any of the A or $\mathrm{A}^{*}$ scans despite there apparently being 15 or fewer movements (sufficient reacquisitions) in 3 of the motion A scans and the $A^{*}$ scan. This could be due to having different segments acquired in regions of different coil sensitivity and field inhomogeneity causing abrupt changes in the k-space data. Different segments within an image could also have varying VEPCASL encoding if movement of the vessels from the initial locations changes the intended inversion efficiency. Looking at the example motion plots in Fig. 2, three categories of points can be identified: 1) large motions which will definitely be reacquired; 2) smaller motions close to the time when a change in position is detected, which will also be reacquired; 3) points on the plateaus where the subject has not moved and will not be reacquired. Therefore it is possible that several category-3 segments, which are acquired in different regions of the coil and $\mathrm{B}_{0}$ field distribution or with different VEPCASL encoding will comprise an image and thus there could be k-space signal differences and discontinuities. In motion conditions $\mathrm{B}$ and $\mathrm{C}$, neighbouring segments will all be reacquired so it is expected that such k-space discontinuities would be less frequent. In addition, the motion $\mathrm{A}$ condition is perhaps a particularly contrived type of movement and is less likely to occur in patient groups. 
Volumetric EPI navigators with $8 \mathrm{~mm}$ isotropic resolution acquired in $275 \mathrm{~ms}$ were implemented in a once-per-segment navigation strategy. These navigators have been shown to provide accurate head position and orientation measurements with $<0.5 \mathrm{~mm}$ jitter $(16,17)$. In this study, the median motion scores in "stationary" phantom and in-vivo scans were $\sim 40 \mu \mathrm{m}$ and $\sim 200 \mu \mathrm{m}$, respectively (see Table 1). These results give some indication of the accuracy of a "true" zero motion measurement and the precision. Note that the in-vivo metrics from these "stationary" scans potentially include physiological and unintentional motion and also that the EPI navigator motion tracking has previously been observed to be object- and contrast-dependent. Therefore, despite the relatively low $8 \mathrm{~mm}$ isotropic spatial resolution, the EPI navigators appear to provide accurate, submillimetre motion information. The Fourier shift theorem can be used to demonstrate that the ultimate accuracy of a motion estimate from a navigator is not necessarily limited by the image resolution. For example, measuring a translation via k-space data requires a linear fit to the phase difference, and the variance of this fit depends on the SNR of the k-space data. In k-space, increasing image resolution will only add low-signal data points at the edges, so the benefit of navigator resolution in this context is not obvious. In addition to the resolution vs. accuracy issue, a recent study showed that even with inaccurate motion estimates (up to $1^{\circ}$ and $6 \mathrm{~mm}$ biases due to incorrect calibration) data quality improves (36). Hence navigator requirements are a complex topic, so it is important that empirically motion correction reduces artifacts and enhances detail, which is particularly clear in retrospective corrections where direct before/after comparisons are available (21).

The native VEPCASL angiography sequence contains no "dead" time in which to place the $275 \mathrm{~ms}$ navigator. The addition of the EPI navigator module after the imaging readout therefore reduced the possible number of dynamic cardiac phases. This was done in order to avoid the acquisition window (imaging segment readout plus navigator duration) extending over a cardiac trigger, which would result in waiting for the next cardiac trigger to begin the acquisition of the next segment - i.e., 
the acquisition of a segment would generally last three heartbeats rather than two. Despite having 2-3 fewer cardiac phases, the current sequence demonstrates the scope for improvement with prospective motion correction. Recent developments have shown that the navigator acquisition can be accelerated with simultaneous multi-slice acquisition to a time of $26 \mathrm{~ms}$ (37), which would clearly be of direct relevance to this and many other PMC applications. Also, previous studies have demonstrated good image quality in the cerebral vessels without cardiac triggering (1). Removing the cardiac triggering would negate the need to reduce the number of acquired cardiac phases, instead the addition of a navigator would increase the scan time by $\sim 17 \%$ (not including extra time for reacquisitions).

The navigator acquisition was placed immediately after the imaging readout of each segment. Hence, all movements between navigators need reacquisition because motion is detected after the segment is acquired, as demonstrated in the phantom experiments of Fig. 2. The preferred placement immediately before the imaging readout was not possible because the readout needs to immediately follow the VEPCASL preparation to enable imaging of blood in the proximal arteries and the navigator acquisition and feedback time would delay the segment readout by 400$500 \mathrm{~ms}$. Having the navigator measurement and update immediately before the imaging readout would correct for movement during the pre-saturation and VEPCASL preparation but the possibility of motion during the readout (and the navigator) remains. Using the motion-score-based reacquisition, it would not be determined whether the motion detected between two navigators was during the imaging readout or during the pre-saturation and VEPCASL preparation. In this angiography sequence, there would be $\sim 50 \%$ probability (based on approximate sequence timings) that a movement that could not be reacquired occurred after the segment readout and hence was detected by the navigator and corrected for. Therefore having a pre-imaging navigator measurement and update is expected to reduce the reliance on reacquisitions. This is assuming that imaging inconsistencies due to head motion have a larger effect on the final angiogram quality than changes in the VEPCASL labelling efficiency due to motion of the neck. The VEPCASL 
labelling plane in the neck is expected to remain relatively still for small- to medium-sized movements of the head and it seems that the major problem is that imaging artifacts can obscure smaller vessels. The effects of VEPCASL encoding and analysis appear to be secondary due to the robustness of the analysis, which allows for some movement of the vessel locations (4).

A combination of the above approach with retrospective k-space correction (22) could potentially be utilised to compensate for the segments that were motioncorrupted but could not be reacquired. The movement parameters detected by the navigator could be used to re-align the segment data in k-space. This correction would be more effective for in-plane rotation and translation compared to throughplane motions. Therefore the reacquisition scheme could be weighted towards movement with a through-plane component so that retrospective correction could effectively correct any residual motion-corrupted segments.

The navigator duration and reacquisition issues discussed above, which are important for once-per-segment MR-based navigation, are of course avoided with an independent tracking system that can apply continuous updates. However, the ease-of-use of MR navigation is attractive for clinical settings and for easier widespread adoption. For the price of a $\sim 25 \%$ increase in scan time a substantial improvement in image quality has been demonstrated.

\section{Conclusions}

This study used $275 \mathrm{~ms}$ EPI navigator acquisitions to measure and prospectively correct motion in a vessel-encoded angiography sequence, which is particularly sensitive to artifacts caused by movement between segments. The technique would also be relevant to non-vessel-encoded angiography sequences using segmented imaging readouts. Due to the positioning of the navigator after the segment readout, reacquisition of motion-corrupted segments was shown to be important for complete correction of imaging artifacts. The navigator acquisition did not affect 
image quality in healthy volunteers and using PMC with reacquisition improved the quality of the angiograms in comparisons with uncorrected scans with similar deliberate motion. The reacquisition of 15 segments during an extra $\sim 30 \mathrm{~s}$ of measurement is expected to be an effective strategy to improve the robustness of the sequence for patient scanning.

\section{Acknowledgements}

We are grateful to the NIHR Oxford Biomedical Research Centre and the Royal Academy of Engineering for funding and for the facilities provided by the Acute Vascular Imaging Centre (AVIC). We thank the following people at AVIC for helpful discussions and assistance with data acquisition: James Kennedy, George Harston, Davide Carone, Juliet Semple and Peter Manley. We also thank the Dunhill Medical Trust for support (PJ).

\section{References}

1. Okell TW, Chappell MA, Woolrich MW, Gunther M, Feinberg DA, Jezzard P. Vesselencoded dynamic magnetic resonance angiography using arterial spin labeling. Magn Reson Med 2010;64:698-706.

2. Robson PM, Dai W, Shankaranarayanan A, Rofsky NM, Alsop DC. Time-resolved vessel-selective digital subtraction MR angiography of the cerebral vasculature with arterial spin labeling. Radiology 2010;257:507-515.

3. Okell TW, Chappell MA, Schulz UG, Jezzard P. A kinetic model for vessel-encoded dynamic angiography with arterial spin labeling. Magn Reson Med 2012;68:969-979.

4. Chappell MA, Okell TW, Payne SJ, Jezzard P, Woolrich MW. A fast analysis method for non-invasive imaging of blood flow in individual cerebral arteries using vesselencoded arterial spin labelling angiography. Med Image Anal 2012;16:831-839.

5. Nakamura M, Yoneyama M, Tabuchi T, Takemura A, Obara M, Tatsuno S, Sawano S. Vessel-selective, non-contrast enhanced, time-resolved MR angiography with vesselselective arterial spin labeling technique (CINEMA-SELECT) in intracranial arteries. Radiol Phys Technol 2013;6:327-334.

6 . Wong EC. Vessel-encoded arterial spin-labeling using pseudocontinuous tagging. Magn Reson Med 2007;58:1086-1091.

7. Liebeskind DS. Collateral circulation. Stroke 2003;34:2279-2284.

8. Friston KJ, Williams S, Howard R, Frackowiak RS, Turner R. Movement-related effects in fMRI time-series. Magn Reson Med 1996;35:346-355. 
9. Jenkinson M, Bannister P, Brady M, Smith S. Improved optimization for the robust and accurate linear registration and motion correction of brain images. Neuroimage 2002; $17: 825-841$.

10. Ward HA, Riederer SJ, Grimm RC, Ehman RL, Felmlee JP, Jack CRJ. Prospective multiaxial motion correction for fMRI. Magn Reson Med 2000;43:459-469.

11. Thesen S, Heid O, Mueller E, Schad LR. Prospective acquisition correction for head motion with image-based tracking for real-time fMRI. Magn Reson Med 2000;44:457465.

12. Welch EB, Manduca A, Grimm RC, Ward HA, Jack CRJ. Spherical navigator echoes for full 3D rigid body motion measurement in MRI. Magn Reson Med 2002;47:32-41. 13. Zaitsev M, Dold C, Sakas G, Hennig J, Speck O. Magnetic resonance imaging of freely moving objects: prospective real-time motion correction using an external optical motion tracking system. Neuroimage 2006;31:1038-1050.

14. van der Kouwe AJW, Benner T, Dale AM. Real-time rigid body motion correction and shimming using cloverleaf navigators. Magn Reson Med 2006;56:1019-1032.

15. White N, Roddey C, Shankaranarayanan A, Han E, Rettmann D, Santos J, Kuperman J, Dale A. PROMO: Real-time prospective motion correction in MRI using image-based tracking. Magn Reson Med 2010;63:91-105.

16. Hess AT, Tisdall MD, Andronesi OC, Meintjes EM, van der Kouwe AJW. Real-time motion and B0 corrected single voxel spectroscopy using volumetric navigators. Magn Reson Med 2011;66:314-323.

17. Tisdall MD, Hess AT, Reuter M, Meintjes EM, Fischl B, van der Kouwe AJW. Volumetric navigators for prospective motion correction and selective reacquisition in neuroanatomical MRI. Magn Reson Med 2012;68:389-399.

18. Atkinson D, Hill DL, Stoyle PN, Summers PE, Keevil SF. Automatic correction of motion artifacts in magnetic resonance images using an entropy focus criterion. IEEE Trans Med Imaging 1997;16:903-910.

19. Pipe JG. An optimized center-out k-space trajectory for multishot MRI: comparison with spiral and projection reconstruction. Magn Reson Med 1999;42:714-720.

20. Bydder M, Larkman DJ, Hajnal JV. Detection and elimination of motion artifacts by regeneration of k-space. Magn Reson Med 2002;47:677-686.

21. Gallichan D, Marques J, Gruetter R. Retrospective correction of involuntary microscopic head movement using highly accelerated fat image navigators (3D FatNavs) at 7T. Magn Reson Med 2015;doi:10.1002/mrm.25670.

22. Maclaren J, Lee KJ, Luengviriya C, Speck O, Zaitsev M. Combined prospective and retrospective motion correction to relax navigator requirements. Magn Reson Med 2011;65:1724-1732.

23. Derbyshire JA, Wright GA, Henkelman RM, Hinks RS. Dynamic scan-plane tracking using MR position monitoring. J Magn Reson Imaging 1998;8:924-932.

24. Ooi MB, Krueger S, Thomas WJ, Swaminathan SV, Brown TR. Prospective real-time correction for arbitrary head motion using active markers. Magn Reson Med 2009;62:943-954.

25. Cheng JY, Alley MT, Cunningham CH, Vasanawala SS, Pauly JM, Lustig M. Nonrigid motion correction in 3D using autofocusing with localized linear translations. Magn Reson Med 2012;68:1785-1797. 
26. Ehman RL, Felmlee JP. Adaptive technique for high-definition MR imaging of moving structures. Radiology 1989;173:255-263.

27. Pipe JG. Motion correction with PROPELLER MRI: application to head motion and free-breathing cardiac imaging. Magn Reson Med 1999;42:963-969.

28. Liu C, Bammer R, Kim D, Moseley ME. Self-navigated interleaved spiral (SNAILS): application to high-resolution diffusion tensor imaging. Magn Reson Med 2004;52:13881396.

29. Fu ZW, Wang Y, Grimm RC, Rossman PJ, Felmlee JP, Riederer SJ, Ehman RL.

Orbital navigator echoes for motion measurements in magnetic resonance imaging. Magn Reson Med 1995;34:746-753.

30. van der Kouwe AJW, Tisdall MD, Bhat H, Gagoski B, Heberlein K. Prospective motion correction of 3D GRASE PASL acquisitions with volume navigators. In: Proceedings of the 21st Annual Meeting of ISMRM, 2013 (abstract 3030).

31. Gunther M, Oshio K, Feinberg DA. Single-shot 3D imaging techniques improve arterial spin labeling perfusion measurements. Magn Reson Med 2005;54:491-498. 32. Grinstead JW, Bhat H, Tisdall MD, van der Kouwe AJW, Rooney W, Laub G. Simultaneous MPRAGE and non-contrast MRA with prospective motion correction using volumetric navigators. In: Proceedings of the 23rd Annual Meeting of ISMRM, 2015 (abstract 3676).

33. Okell TW, Schmitt P, Bi X, Chappell MA, Tijssen RHN, Miller KL, Jezzard P. 4D vessel-encoded arterial spin labeling angiography. In: Proceedings of the 19th Annual Meeting of ISMRM, 2011 (abstract 4034).

34. Griswold MA, Jakob PM, Heidemann RM, Nittka M, Jellus V, Wang J, Kiefer B, Haase A. Generalized autocalibrating partially parallel acquisitions (GRAPPA). Magn Reson Med 2002;47:1202-1210.

35. Okell TW, Chappell MA, Kelly ME, Jezzard P. Cerebral blood flow quantification using vessel-encoded arterial spin labeling. J Cereb Blood Flow Metab 2013;33:17161724.

36. Maclaren J, Aksoy M, Zahneisen B, Bammer R. Camera placement for optical prospective motion correction: mechanical tolerance analysis. In: Proceedings of the 23rd Annual Meeting of ISMRM, 2015 (abstract 3669).

37. Bhat H, Tisdall MD, Cauley SF, Witzel T, Setsompop K, van der Kouwe AJW, Heberlein K. Simultaneous multi-slice (SMS) accelerated EPI navigators for prospective motion correction in the brain. In: Proceedings of the 23rd Annual Meeting of ISMRM, 2015 (abstract 817).

\section{Figures}

Figure 1: Sequence diagram of the VEPCASL angiography sequence with navigator acquisition inserted after the imaging readout and position and orientation updates (from the previous navigator measurement) before the imaging readout. A $10 \mathrm{~ms}$ pause was required to update the imaging coordinates. $\mathrm{N}$ cardiac phases (typically 6-9 in the original sequence) are acquired for each segment, which consisted of 10 $\mathrm{k}$-space lines in this study. 
Figure 2: Examples of the three motion conditions used in the deliberate motion experiments. The plots of the three rotations and three translations detected from each navigator image are shown in the left-hand column as well as the corresponding motion scores in the right-hand column.

Figure 3: Phantom experiments demonstrating the effect of motion between segments for rotation about the $\mathrm{z}$ (slice) axis (top row) and $\mathrm{z}$ translation (bottom row). A scan acquired without motion was compared to scans acquired with repeated motion (see motion logs in Sup. Fig. S2) using no correction (PMC off), PMC but no reacquisition and $\mathrm{PMC}$ with reacquisition of 1 segment.

Figure 4: In-vivo experiments comparing the original VEPCASL angiography sequence (left column) to the sequence with navigators (right column) in subjects 2 and 4. The angiograms were acquired without deliberate motion. The temporal means of the cardiac phases are shown. Colour code: $\mathrm{RICA}=$ red, LICA $=$ green, RVA $=$ blue, $\mathrm{LVA}=$ purple.

Figure 5: In-vivo angiograms from the motion experiments comparing PMC off to PMC on with 15 reacquisitions. Motion conditions A (discrete, large movements), B (continuous, small movements) and C (continuous, large movements) are compared to scans without deliberate motion. The temporal means of the cardiac phases are shown. Colour code: RICA = red, LICA = green, RVA = blue, LVA = purple.

Figure 6: Motion experiment summaries for all comparisons in all four subjects of the number of motions, the mean motion scores of the significant motions and the image quality scores. (a) Number of motions detected using the $0.5 \mathrm{~mm}$ motion score threshold. The red dotted line marks the 15 reacquisitions used in the motion experiments. (b) Mean motion score of the scores above the $0.5 \mathrm{~mm}$ threshold. (c) Image quality scores based on the visibility of vessels relative to the angiogram acquired without motion. The red circles mark the scans with PMC and reacquisition that had more than 15 significant motions (i.e., above the red line in panel (a)).

Figure 7: In-vivo data showing the effect of retrospectively removing reacquisitions from the reconstruction. Using all 15 reacquisitions is compared to using the first 10 , the first 5 and none. Data from motion conditions $A, B$ and $C$ in subject 1 are shown. Colour code: RICA = red, LICA = green, RVA = blue, $L V A=$ purple.

Figure 8: In-vivo motion experiments with fewer than 15 movements. PMC off was compared to PMC on with 15 reacquisitions. (a) Angiograms from scans during motion conditions $\mathrm{A}^{*}, \mathrm{~B}^{*}$ and $\mathrm{C}^{*}$ are compared to a scan without deliberate motion. (b) The detected translation and rotation plots from the scans PMC off (top row) and PMC on with 15 reacquisitions (bottom row) being compared. Colour code: RICA $=$ red, $L I C A=$ green, RVA = blue, $L V A=$ purple. 
Supporting Figure S1: Motion plots from an in-vivo scan without intentional motion (subject 1).

Supporting Figure S2: Motion plots from the phantom experiments testing motion between segments. The plots show similar motion between the compared scans with PMC off, PMC on but no reacquisition and PMC on and one reacquisition.

Supporting Figure S3: Motion summaries of the additional experiment with shorter motion periods. Top row: Number of motions detected using the $0.5 \mathrm{~mm}$ motion score threshold. The red dotted line marks the 15 reacquisitions used in the motion experiments. Bottom row: Mean motion score of the scores above the $0.5 \mathrm{~mm}$ threshold. 


\section{Table 1}

\begin{tabular}{lccccccc} 
Scan & \multicolumn{9}{c}{ Temporal standard deviations of rigid-body motion estimates } & Median motion \\
& $\Delta_{x}(\mu \mathrm{m})$ & $\Delta_{y}(\mu \mathrm{m})$ & $\Delta_{z}(\mu \mathrm{m})$ & $\theta_{x}\left(10^{-3}\right)$ & $\theta_{y}\left(10^{-3}\right)$ & $\theta_{z}\left(10^{-3}\right)$ & score $(\mu \mathrm{m})$ \\
\hline Phantom 1 & 6.8 & 9.4 & 13.6 & 5.9 & 5.7 & 8.5 & 35.4 \\
Phantom 2 & 6.8 & 19.4 & 7.0 & 20.3 & 3.3 & 15.8 & 48.0 \\
Subject 1 & 93.7 & 53.3 & 79.0 & 25.4 & 48.1 & 22.8 & 166.9 \\
Subject 2 & 98.2 & 47.7 & 70.4 & 54.8 & 75.1 & 18.6 & 218.8 \\
Subject 3 & 168.9 & 68.6 & 141.0 & 68.1 & 191.0 & 45.0 & 238.3 \\
Subject 4 & 87.6 & 38.5 & 48.4 & 39.8 & 57.4 & 18.0 & 144.1
\end{tabular}

The phantom and in-vivo scans consisted of 7 and 70 motion estimates, respectively. 
Figure 1: Sequence diagram of the VEPCASL angiography sequence with navigator acquisition inserted after the imaging readout and position and orientation updates (from the previous navigator measurement) before the imaging readout. A $10 \mathrm{~ms}$ pause was required to update the imaging coordinates. $\mathrm{N}$ cardiac phases (typically 6-9 in the original sequence) are acquired for each segment, which consisted of $10 \mathrm{k}$-space lines in this study.

$22 \times 8 \mathrm{~mm}(300 \times 300 \mathrm{DPI})$ 
motion A
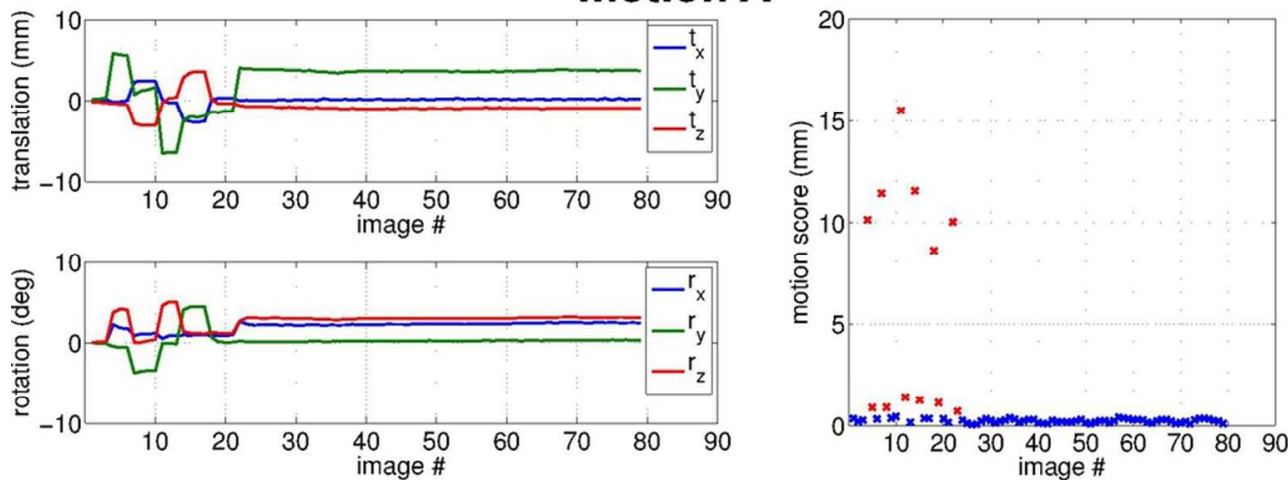

motion B
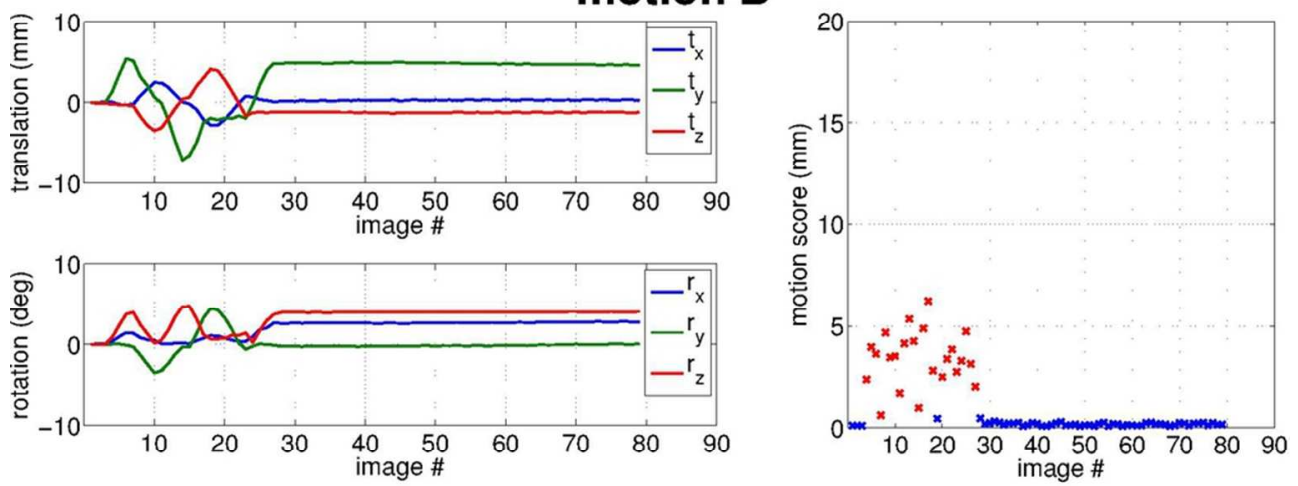

motion C
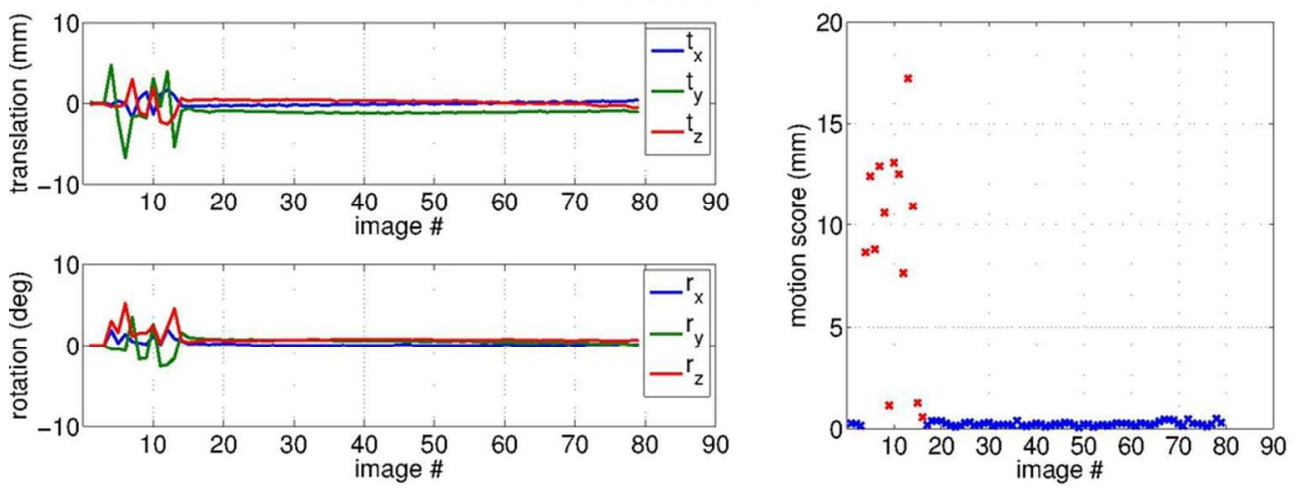

Figure 2: Examples of the three motion conditions used in the deliberate motion experiments. The plots of the three rotations and three translations detected from each navigator image are shown in the left-hand column as well as the corresponding motion scores in the right-hand column.

$85 \times 103 \mathrm{~mm}(300 \times 300$ DPI $)$ 
Figure 3: Phantom experiments demonstrating the effect of motion between segments for rotation about the $z$ (slice) axis (top row) and $z$ translation (bottom row). A scan acquired without motion was compared to scans acquired with repeated motion (see motion logs in Sup. Fig. 2) using no correction (PMC off), PMC but no reacquisition and $\mathrm{PMC}$ with reacquisition of 1 segment. $78 \times 38 \mathrm{~mm}(300 \times 300 \mathrm{DPI})$ 


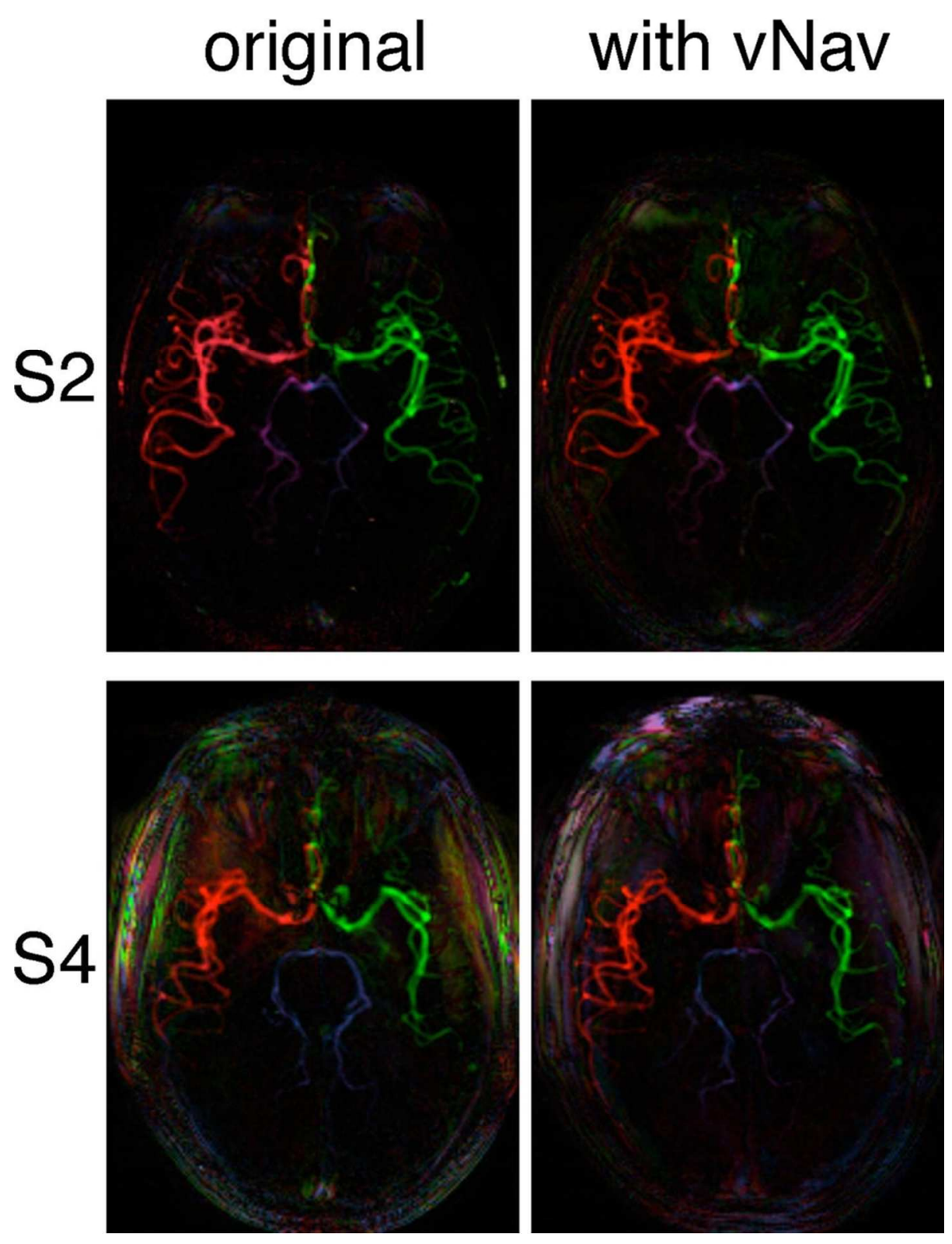

Figure 4: In-vivo experiments comparing the original VEPCASL angiography sequence (left column) to the sequence with navigators (right column) in subjects 2 and 4 . The angiograms were acquired without deliberate motion. The temporal means of the cardiac phases are shown. Colour code: RICA = red, LICA = green, RVA = blue, LVA = purple. $73 \times 96 \mathrm{~mm}(300 \times 300 \mathrm{DPI})$ 
motion C

PMC off PMC on PMC off PMC on

\section{PMC off PMC on}

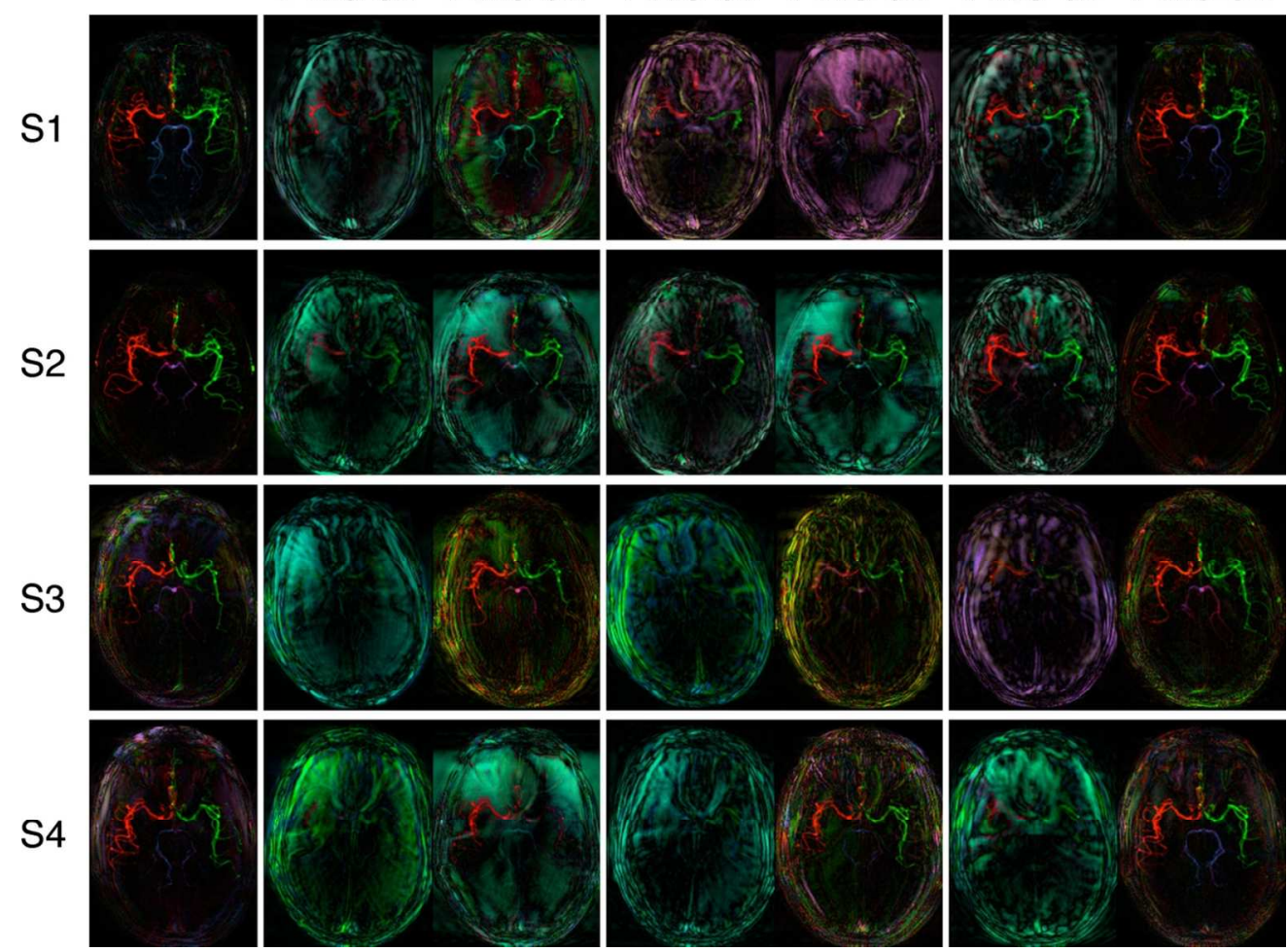

Figure 5: In-vivo angiograms from the motion experiments comparing PMC off to PMC on with 15 reacquisitions. Motion conditions A (discrete, large movements), B (continuous, small movements) and C (continuous, large movements) are compared to scans without deliberate motion. The temporal means of the cardiac phases are shown. Colour code: RICA $=$ red, LICA $=$ green, RVA $=$ blue, LVA $=$ purple. $114 \times 92 \mathrm{~mm}(300 \times 300 \mathrm{DPI})$ 
Figure 6: Motion experiment summaries for all comparisons in all four subjects of the number of motions, the mean motion scores of the significant motions and the image quality scores. (a) Number of motions detected using the $0.5 \mathrm{~mm}$ motion score threshold. The red dotted line marks the 15 reacquisitions used in the motion experiments. (b) Mean motion score of the scores above the $0.5 \mathrm{~mm}$ threshold. (c) Image quality scores based on the visibility of vessels relative to the angiogram acquired without motion. The red circles mark the scans with PMC and reacquisition that had more than 15 significant motions (i.e., above the red line in panel (a)).

$59 \times 22 \mathrm{~mm}(300 \times 300 \mathrm{DPI})$ 


\section{\# reacqs used in recon (S1)}

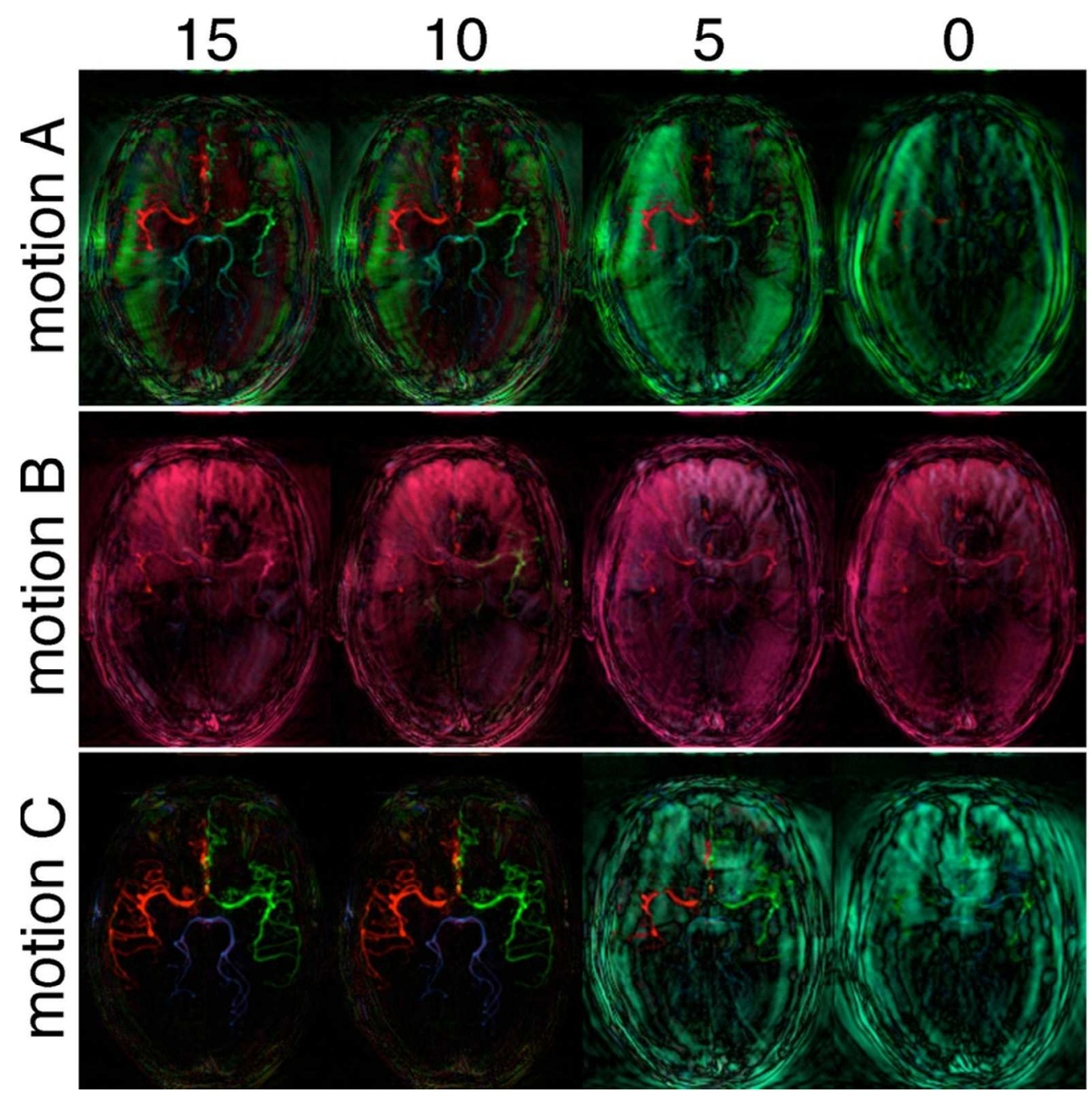

Figure 7: In-vivo data showing the effect of retrospectively removing reacquisitions from the reconstruction. Using all 15 reacquisitions is compared to using the first 10, the first 5 and none. Data from motion conditions A, B and C in subject 1 are shown. Colour code: RICA = red, LICA = green, RVA = blue, LVA = purple.

$80 \times 86 \mathrm{~mm}(300 \times 300$ DPI $)$ 


\section{a} no motion motion $\mathrm{A}^{*}$ motion $\mathrm{B}^{*}$ motion $\mathrm{C}^{*}$

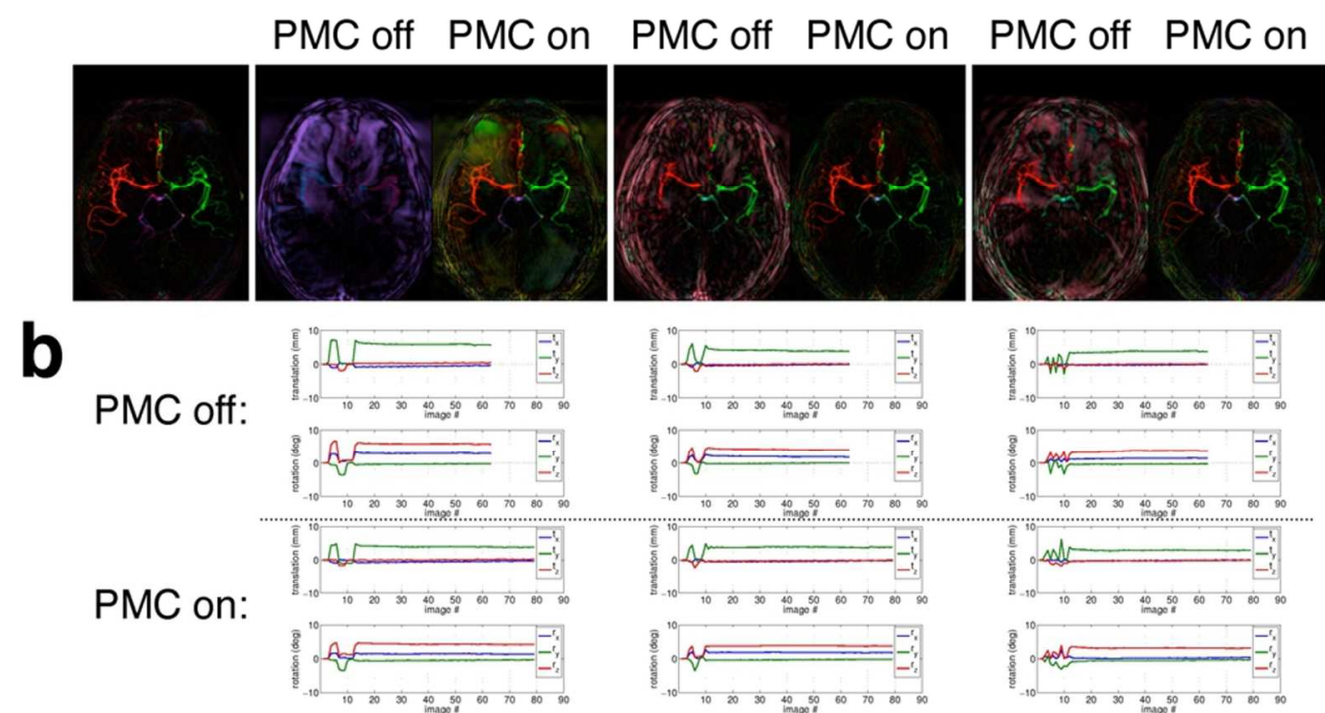

Figure 8: In-vivo motion experiments with fewer than 15 movements. PMC off was compared to PMC on with 15 reacquisitions. (a) Angiograms from scans during motion conditions $A^{*}, B^{*}$ and $C^{*}$ are compared to a scan without deliberate motion. (b) The detected translation and rotation plots from the scans PMC off (top row) and PMC on with 15 reacquisitions (bottom row) being compared. Colour code: RICA = red, LICA = green, RVA = blue, LVA = purple. $84 \times 51 \mathrm{~mm}(300 \times 300 \mathrm{DPI})$ 

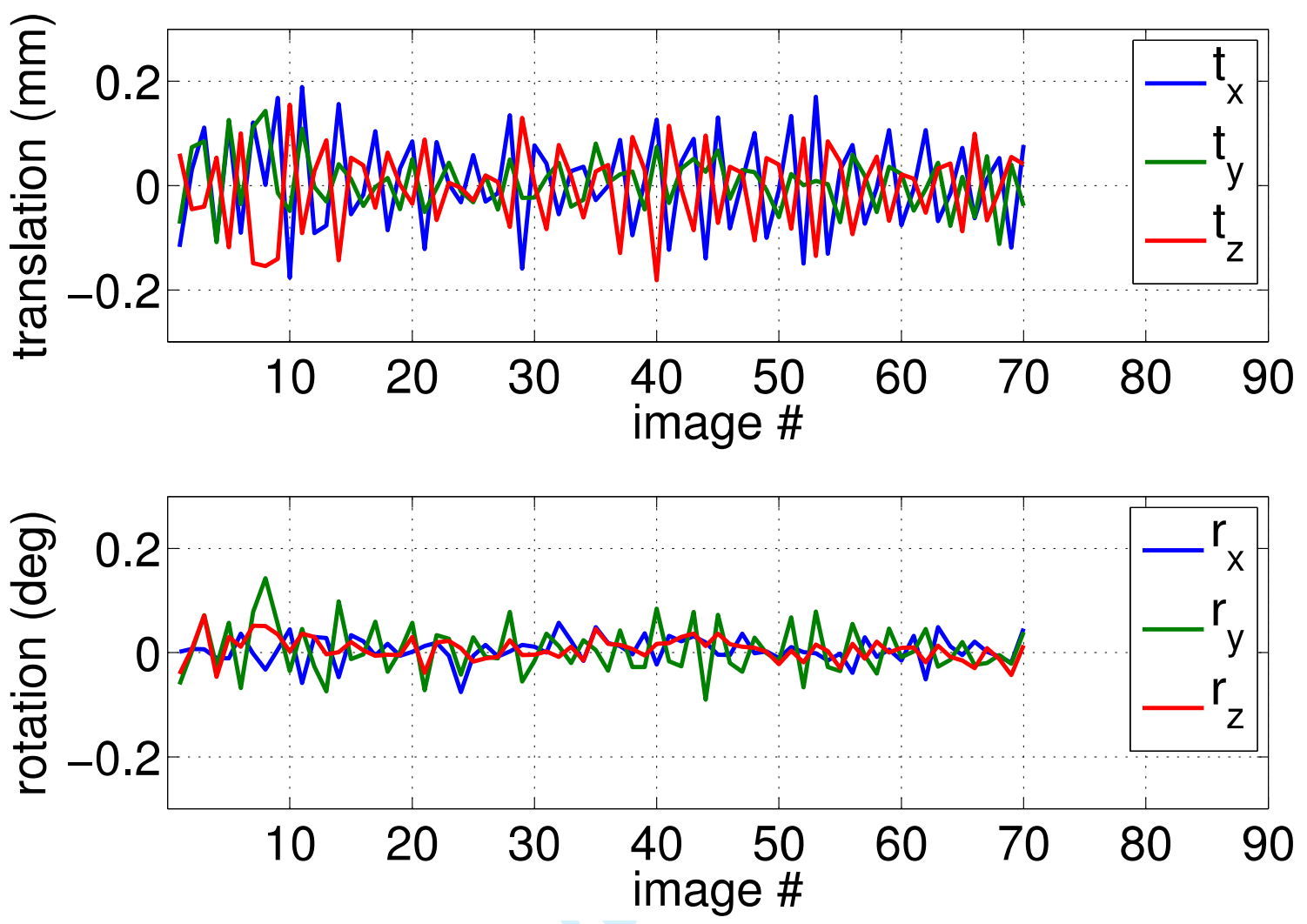

Supporting Figure S1: motion plots from an in-vivo scan without intentional motion (subject 1).

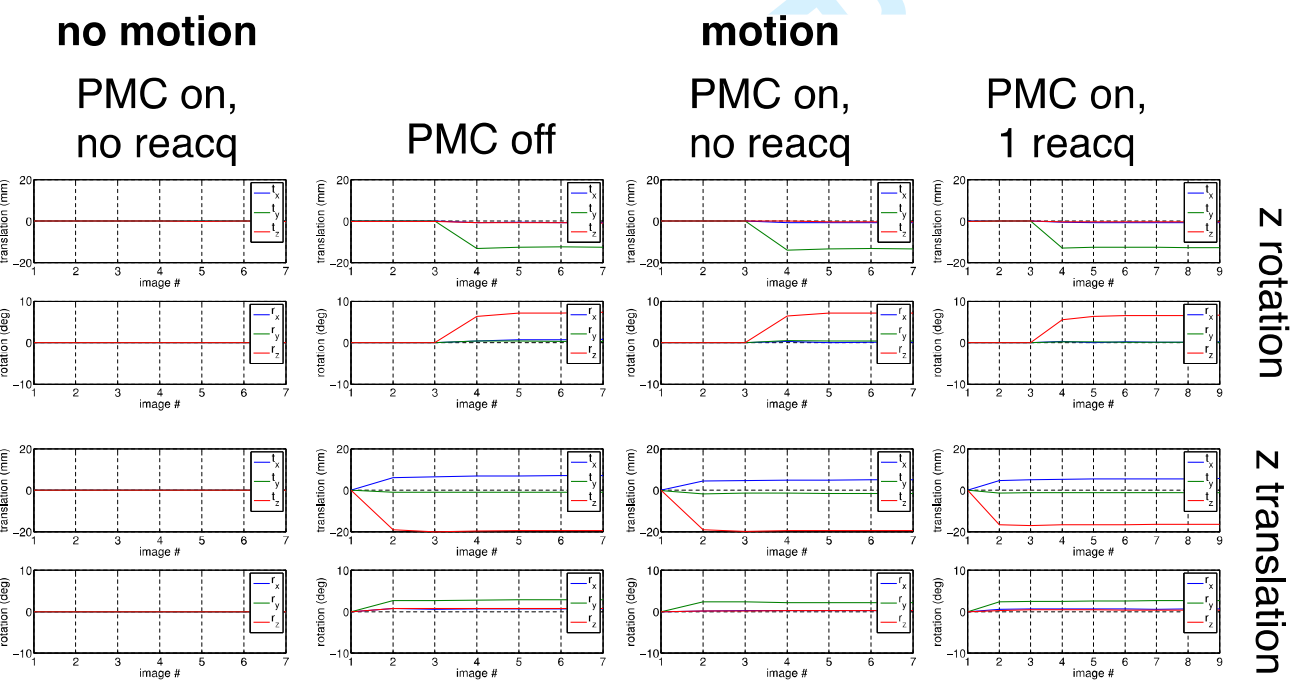

Supporting Figure S2: Motion plots from the phantom experiments testing motion between segments. The plots show similar motion between the compared scans with PMC off, PMC on but no reacquisition and PMC on and one reacquisition. 

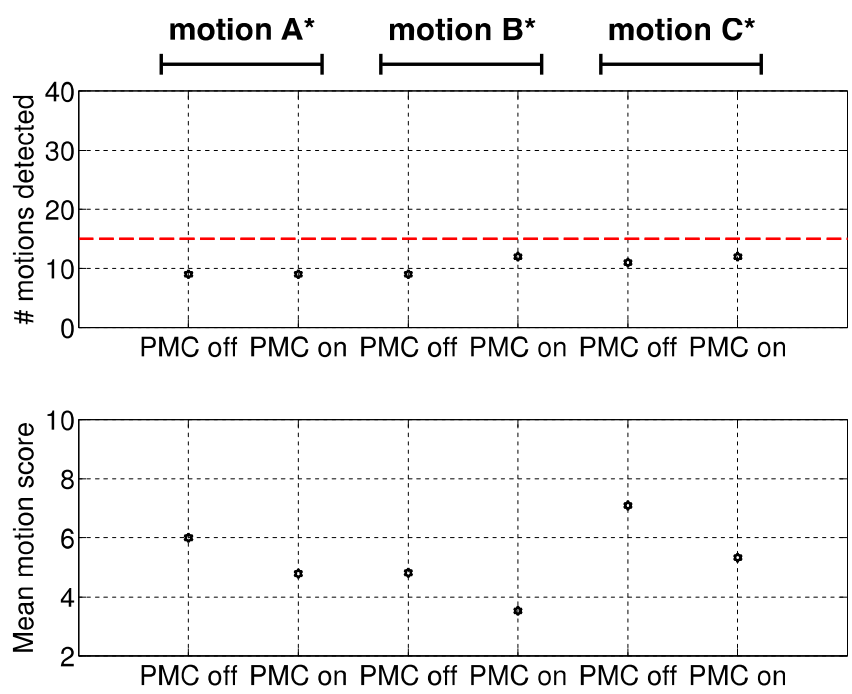

Supporting Figure S3: Motion summaries of the additional experiment with shorter motion periods. Top row: Number of motions detected using the $0.5 \mathrm{~mm}$ motion score threshold. The red dotted line marks the 15 reacquisitions used in the motion experiments. Bottom row: Mean motion score of the scores above the $0.5 \mathrm{~mm}$ threshold. 